\title{
Induced current in high-dimensional AdS spacetime in the presence of a cosmic string and a compactified extra dimension
}

\author{
W. Oliveira dos Santos, ${ }^{*}$ H. F. Mota, ${ }^{\dagger}$ and E. R. Bezerra de Mello \\ Departamento de Física, Universidade Federal da Paraíba 58059-900, \\ Caixa Postal 5008, João Pessoa, Paraíba, Brazil
}

(Received 10 September 2018; published 14 February 2019)

\begin{abstract}
In this paper, we analyze the bosonic current densities induced by a magnetic flux running along the core of an idealized cosmic string in a high-dimensional anti-de Sitter spacetime, admitting that an extra dimension coordinate is compactified. Additionally, we admit the presence of a magnetic flux enclosed by the compactified axis. To develop this analysis, we calculate the complete set of normalized bosonic wave functions obeying a quasiperiodicity condition, with arbitrary phase $\beta$, along the compactified extra dimension. In this context, only azimuthal and axial currents densities take place. As to the azimuthal current, two contributions appear. The first one corresponds to the standard azimuthal current in highdimensional anti-de Sitter spacetime with a cosmic string without compactification, while the second contribution is a new one, induced by the compactification itself. The latter is an even function of the magnetic flux enclosed by the compactified axis and is an odd function of the magnetic flux along its core with period equal to quantum flux, $\Phi_{0}=2 \pi / e$. On the other hand, the nonzero axial current density is an even function of the magnetic flux along the core of the string and an odd function of the magnetic flux enclosed by the compactified axis. We also find that the axial current density vanishes for untwisted and twisted bosonic fields in the absence of the magnetic flux enclosed by the compactified axis. Some asymptotic expressions for the current density are provided for specific limiting cases of the physical parameter of the model.
\end{abstract}

DOI: 10.1103/PhysRevD.99.045005

\section{INTRODUCTION}

The physics underlying quantum vacuum fluctuations arises once quantum aspects of relativistic phenomena are taken into account. That means a quantized relativistic field (scalar, electromagnetic, or fermionic) will have a fluctuating ground state. In Minkowski spacetime, for instance, the vacuum expectation value (VEV) of physical observables, as a consequence of quantum vacuum fluctuations of relativistic fields, is zero unless the vacuum is somehow "perturbed" by external influences. These external influences are in general boundary conditions of some sort or coupled external fields. One very known physical observable that gets a nonzero VEV under external influences is the energy density that characterizes the Casimir effect [1-3]. Another physical observable of

\footnotetext{
*wagner.physics@gmail.com

†hmota@fisica.ufpb.br

*emello@fisica.ufpb.br
}

Published by the American Physical Society under the terms of the Creative Commons Attribution 4.0 International license. Further distribution of this work must maintain attribution to the author(s) and the published article's title, journal citation, and DOI. Funded by SCOAP ${ }^{3}$. interest that averages to a nonzero value under these circumstances is the four-current density due to charged fields. This is of special importance since the VEV of the four-current density can provide a better understanding of the dynamics of the electromagnetic field once it is used as a source in the semiclassical Maxwell equations.

An additional feature related to modifications of quantum vacuum fluctuations of relativistic fields is its occurrence faces a curved background. It has been known that geometrical and topological aspects of a curved spacetime also induce a nonzero VEV of physical observables [1-3]. In particular, the induced VEV of the four-current density by curved backgrounds has been investigated in Refs. [4-11]. Among these curved backgrounds, the anti-de Sitter (AdS) spacetime carries very interesting properties that provide strong motivation to study it [12-17].

By considering a negative cosmological constant, the AdS spacetime is obtained as a solution of Einstein's equations and thus is characterized by a constant negative curvature. Thereby, from a theoretical and fundamental point of view, the AdS spacetime makes it possible to solve several problems exactly as a consequence of its high symmetry, allowing the quantization of fields more easily, besides offering better insight into the quantization of fields in other curved spacetimes. Moreover, the AdS spacetime 
arises as a ground-state solution of string and supergravity theories and also appears in the context of AdS/CFT correspondence, a scenario that makes possible the realization of the holographic principle, relating string theory (supergravity) in a high-dimensional AdS spacetime with a conformal field theory constructed in its boundary [18]. In addition, the AdS background geometry is relevant in branewold scenarios with large extra dimensions, offering a way to solve the hierarchy problem between the gravitational and electroweak mass scales [19].

The presence of a cosmic string in AdS spacetime provides more interesting phenomena, since this combined geometry makes it possible to identify in the VEV of some observable, the contributions come from either parts, namely, from the AdS geometry and cosmic string topology. Cosmic strings are linear topological defects that are predicted in the context of both gauge field theories and supersymmetric extensions of the Standard Model of particle physics, as well as in the context of string theory [20-25]. The spacetime of a straight, infinitely long, and structureless cosmic string is characterized by a conical topology arising due to the angle deficit in the plane perpendicular to it [20-22]. Phenomenologically, current observations of $\mathrm{CMB}$ suggest cosmic strings can contribute to a small percentage of the primordial density perturbations [26] in the Universe and can also play a important role in other cosmological, astrophysical, and gravitational phenomena [22-25].

In the present paper, we are interested in calculating the VEV of the current density associated with a charged scalar field and investigate the effects arising from the geometry and topology of a high-dimensional AdS spacetime in the presence of a cosmic string carrying a magnetic flux. In addition, we will assume a compactification of one extra dimension and the existence of a constant vector potential along it. Thus, the presence of these magnetic fluxes as well as the compactified extra dimension will also provide additional contributions to the VEV of the current density, as we shall see.

The presence of extra compact dimensions is a characteristic aspect of all the theories mentioned before in which the AdS spacetime plays a key role and, as previously said, induces nonzero contributions to physical observables such as the energy-momentum tensor, which has not only the energy density component but also the stresses components (see Ref. [5] and references therein). In this case, for instance, the vacuum energy density induced by the extra compact dimensions offers an explanation for the observed and still unexplained accelerated expansion of the Universe. In Kaluza-Klein-type models and in braneworld scenarios, on the other hand, the dependence of the size of the compact extra dimension by the vacuum energy density serves as a mechanism to stabilize fields known as moduli fields.

This paper is organized as follows. In Sec. II, we present the high-dimensional AdS spacetime in the presence of a cosmic string and obtain the complete set of normalized solutions of the Klein-Gordon equation associated with a charged scalar field in this background, considering the presence of an azimuthal and axial vector potentials. This solution is then submitted to a nontrivial boundary condition that compactifies an extra dimension. This set of solution is used to construct the Wightman function. In Sec. III, we first prove that the VEVs of the charge density, radial current density, and current density associated with the extra dimensions, except the one that is compactifed, are all zero. Finally, the rest of Sec. III is devoted to computing the nonzero azimuthal current density and the nonzero current density associated with the compactified extra dimension. In this case, we show that the azimuthal current density has a pure contribution due to the high-dimensional AdS spacetime with a cosmic string plus a second contribution due to the compactification of the extra dimension. Moreover, we also show that the current density associated with the compactified extra dimension has only the contribution due to the compactification. Section IV is devoted to the main conclusions about our results. Throughout the paper, we use natural units $G=\hbar=c=1$.

\section{KLEIN-GORDON EQUATION AND WIGHTMAN FUNCTION}

The main objective of this section is to obtain the positive frequency Wightman function associated with a massive scalar field in a $(D+1)$-dimensional AdS spacetime, with $D>3$, in the presence of a cosmic string and a compactified extra dimension. This function is important in the calculation of vacuum polarization effects. To do that, we first obtain the complete set of normalized mode functions for the Klein-Gordon equation admitting an arbitrary curvature coupling parameter.

In cylindrical coordinates, the geometry associated with a cosmic string in a $(3+1)$-dimensional AdS spacetime is given by the line element below (considering a static string along the $y$ axis),

$$
d s^{2}=e^{-2 y / a}\left[d t^{2}-d r^{2}-r^{2} d \phi^{2}\right]-d y^{2},
$$

where $r \geq 0$ and $\phi \in[0,2 \pi / q]$ define the coordinates on the conical geometry, $(t, y) \in(-\infty, \infty)$, and the parameter $a$ determines the curvature scale of the background spacetime. The parameter $q \geq 1$ codifies the presence of the cosmic string. Using the Poincaré coordinate defined by $w=a e^{y / a}$, the line element above is written in the form conformally related to the line element associated with a cosmic string in Minkowski spacetime:

$$
d s^{2}=\left(\frac{a}{w}\right)^{2}\left[d t^{2}-d r^{2}-r^{2} d \phi^{2}-d w^{2}\right]
$$

For the new coordinate, one has $w \in[0, \infty)$. Specific values for this coordinates deserve to be mentioned: 
$w=0$ and $w=\infty$ correspond to the AdS boundary and horizon, respectively.

For an idealized cosmic string, i.e., an infinitely thin and long straight cosmic string in the background of Minkowski spacetime, the line element expression inside the brackets of the right-hand side of (2.2), has been derived in Ref. [27] by making use of two approximations: the weak-field approximation and the thin-string one. In this case, the parameter $q$ is related to the mass per unit length $\mu$ of the string by the formula $1 / q=1-4 G \mu$, where $G$ is Newton's gravitational constant. However, the validity of the line element with the planar angle deficit has been extended beyond linear perturbation theory by Refs. [20,28]. In this case, the parameter $q$ does not need to be close to 1 . Note that in braneworld scenarios based on AdS spacetime, to which the results given in this paper could be applied, the fundamental Planck scale is much smaller than $m_{\mathrm{Pl}}$ and can be of order of the string formation energy scale.

The generalization of (2.2) to $(D+1)$-dimensional AdS spacetimes is done in the usual way, by adding extra Euclidean coordinates [13]:

$d s^{2}=\left(\frac{a}{w}\right)^{2}\left[d t^{2}-d r^{2}-r^{2} d \phi^{2}-d w^{2}-\sum_{i=4}^{D}\left(d x^{i}\right)^{2}\right]$.

The Euclidean version of the line element expressed inside the bracket of the above equation has been presented in Ref. [29] and called conical-type line singularity in an arbitrary dimension; therefore, we consider the line element inside (2.3) as a Minkowski version of the cosmic string metric spacetime for higher dimensions. Moreover, a discussion about the generalization of the cosmic string spacetime can also be found in Ref. [30].

The curvature scale $a$ in (2.3) is related to the cosmological constant, $\Lambda$, and the Ricci scalar, $R$, by the formulas

$$
\Lambda=-\frac{D(D-1)}{2 a^{2}}, \quad R=-\frac{D(D+1)}{a^{2}} .
$$

The analysis of the induced current density for a charged massive scalar field in the AdS space described in Poincare coordinates with toroidally compact dimensions has been developed in Ref. [5]. In the latter, it is assumed that, in addition to compact dimensions, the field obeys periodicity conditions with general phases. Moreover, the presence of constant vector potentials has also been considered.

In this present paper, we are interested in calculating the induced vacuum current density, $\left\langle j_{\mu}\right\rangle$, associated with a charged scalar quantum field, $\varphi(x)$, in the cosmic string spacetime in the AdS bulk induced by the presence of magnetic flux running along the string's core. Moreover, we also assume the compactification along only one extra coordinate, defined by $z$ in the expression below: $d s^{2}=\left(\frac{a}{w}\right)^{2}\left[d t^{2}-d r^{2}-r^{2} d \phi^{2}-d w^{2}-d z^{2}-\sum_{i=5}^{D}\left(d x^{i}\right)^{2}\right]$.

Note that we will also consider the presence of a constant vector potential along the extra compact dimension. This compactification is implemented by assuming that $z \in[0, L]$, and the matter field obeys the quasiperiodicity condition

$\varphi\left(t, r, \phi, w, z+L, x^{5}, \ldots, x^{D}\right)=e^{2 \pi i \beta} \varphi\left(t, r, \phi, w, z, x^{5}, \ldots, x^{D}\right)$,

where $0 \leq \beta \leq 1$. The special cases $\beta=0$ and $\beta=1 / 2$ correspond to the untwisted and twisted fields, respectively, along the $z$ direction.

The field equation that governs the quantum dynamics of a charged bosonic field with mass $m$, in a curved background and in the presence of an electromagnetic potential vector, $A_{\mu}$, reads

$$
\left(g^{\mu \nu} D_{\mu} D_{\nu}+m^{2}+\xi R\right) \varphi(x)=0,
$$

with $D_{\mu}=\nabla_{\mu}+i e A_{\mu}$. In addition, we have considered the presence of a nonminimal coupling, $\xi$, between the field and the geometry represented by the Ricci scalar, $R$. Two specific values for the curvature coupling are $\xi=0$ and $\xi=\frac{D-1}{4 D}$, which correspond to minimal and conformal coupling, respectively. Also, we shall assume the existence of the constant vector potentials

$$
A_{\mu}=\left(0,0, A_{\phi}, 0, A_{z}, 0, \ldots, 0\right),
$$

with $A_{\phi}=-q \Phi_{\phi} /(2 \pi)$ and $A_{z}=-\Phi_{z} / L$, with $\Phi_{\phi}$ and $\Phi_{z}$ being the corresponding magnetic fluxes. In quantum field theory, the condition (2.6) changes the spectrum of the vacuum fluctuations compared with the case of uncompactified dimensions and, as a consequence, the induced vacuum current density changes.

In the spacetime defined by (2.5) and in the presence of the vector potentials given above, Eq. (2.7) becomes

$$
\begin{aligned}
& {\left[\frac{\partial^{2}}{\partial t^{2}}-\frac{\partial^{2}}{\partial r^{2}}-\frac{1}{r} \frac{\partial}{\partial r}-\frac{1}{r^{2}}\left(\frac{\partial}{\partial \phi}+i e A_{\phi}\right)^{2}-\left(\frac{\partial}{\partial z}+i e A_{z}\right)^{2}\right.} \\
& \left.-\frac{\partial^{2}}{\partial w^{2}}-\frac{(1-D)}{w} \frac{\partial}{\partial w}+\frac{M(D, m, \xi)}{w^{2}}-\sum_{i=5}^{D} \frac{\partial^{2}}{\partial\left(x^{i}\right)^{2}}\right] \varphi(x)=0,
\end{aligned}
$$

where $M(D, m, \xi)=a^{2} m^{2}-\xi D(D+1)$.

The equation above is completely separable, and its positive energy and regular solution at the origin are given by 
$\varphi(x)=C w^{\frac{D}{2}} J_{\nu}(p w) J_{q|n+\alpha|}(\lambda r) e^{-i E t+i q n \phi+i k_{z} z+i \vec{k} \cdot \vec{x}_{\|}}$.

In the expression above, $\vec{x}_{\|}$represents the coordinates along the $(D-4)$ extra dimensions, and $\vec{k}$ represents the corresponding momentum. Moreover,

$$
\begin{aligned}
\nu & =\sqrt{\frac{D^{2}}{4}+a^{2} m^{2}-\xi D(D+1)}, \\
E & =\sqrt{\lambda^{2}+p^{2}+\vec{k}^{2}+\left(k_{z}+e A_{z}\right)^{2}}, \\
\alpha & =\frac{e A_{\phi}}{q}=-\frac{\Phi_{\phi}}{\Phi_{0}},
\end{aligned}
$$

with $\Phi_{0}=\frac{2 \pi}{e}$, the quantum flux. In (2.10), $J_{\mu}(z)$ represents the Bessel function [31].

The quasiperiodicity condition (2.6) provides a discretization of the quantum number $k_{z}$ as shown below:

$k_{z}=k_{l}=\frac{2 \pi}{L}(l+\beta), \quad$ with $\quad l=0, \pm 1, \pm 2, \ldots$

Therefore,

$$
E=E_{l}=\sqrt{\lambda^{2}+p^{2}+\vec{k}^{2}+\tilde{k}_{l}^{2}},
$$

where

$$
\begin{aligned}
\tilde{k}_{l} & =\frac{2 \pi}{L}(l+\tilde{\beta}), \\
\tilde{\beta} & =\beta+\frac{e A_{z} L}{2 \pi}=\beta-\frac{\Phi_{z}}{\Phi_{0}} .
\end{aligned}
$$

The constant $C$ in (2.10) can be obtained by the normalization condition

$$
\int d^{D} x \sqrt{|g|} g^{00} \varphi_{\sigma^{\prime}}^{*}(x) \varphi_{\sigma}(x)=\frac{1}{2 E} \delta_{\sigma, \sigma^{\prime}},
$$

where the delta symbol on the right-hand side is understood as the Dirac delta function for the continuous quantum number, $\lambda, p$, and $\vec{k}$, and Kronecker delta for the discrete ones, $n$ and $k_{l}$. From (2.15), one finds

$$
|C|=\sqrt{\frac{q a^{1-D} \lambda p}{2 E(2 \pi)^{D-3} L}} .
$$

So, the normalized bosonic wave function reads

$$
\begin{aligned}
\varphi_{\sigma}(x)= & \sqrt{\frac{q a^{1-D} \lambda p}{2 E(2 \pi)^{D-3} L}} w^{\frac{D}{2}} J_{\nu}(p w) J_{q|n+\alpha|}(\lambda r) \\
& \times e^{-i E_{l} t+i q n \phi+i k_{l} z+i \vec{k} \cdot \vec{x}_{\|}} .
\end{aligned}
$$

The properties of the vacuum state can be given by the positive frequency Wightman function,
$W\left(x, x^{\prime}\right)=\left\langle 0\left|\hat{\varphi}(x) \hat{\varphi}^{*}\left(x^{\prime}\right)\right| 0\right\rangle$, where $|0\rangle$ stands for the vacuum state with respect to the observer placed at rest with respect to the string. To evaluate it, we use the mode sum formula

$$
W\left(x, x^{\prime}\right)=\sum_{\sigma} \varphi_{\sigma}(x) \varphi_{\sigma}^{*}\left(x^{\prime}\right)
$$

Substituting (2.17) into (2.18), we obtain

$$
\begin{aligned}
W\left(x, x^{\prime}\right)= & \frac{q a^{1-D}\left(w w^{\prime}\right)^{\frac{D}{2}}}{2(2 \pi)^{D-3} L} \\
& \times \sum_{n=-\infty}^{\infty} e^{i n q \Delta \phi} \sum_{l=-\infty}^{\infty} \int d \vec{k} \int_{0}^{\infty} d p p \int_{0}^{\infty} d \lambda \lambda \\
& \times J_{q|n+\alpha|}(\lambda r) J_{q|n+\alpha|}\left(\lambda r^{\prime}\right) J_{\nu}(p w) J_{\nu}\left(p w^{\prime}\right) \\
& \times \frac{e^{-i E_{l} \Delta t+i k_{l} \Delta z+i \vec{k} \cdot \Delta \vec{x}_{\|}}}{E_{l}}
\end{aligned}
$$

where $\quad \Delta t=t-t^{\prime}, \quad \Delta \phi=\phi-\phi^{\prime}, \quad \Delta z=z-z^{\prime} \quad$ and $\Delta \vec{x}_{\|}=\vec{x}_{\|}-\vec{x}_{\|}^{\prime}$.

To develop the summation over the quantum number $l$, we shall apply the Abel-Plana summation formula [32], which is given by

$$
\begin{aligned}
& \sum_{l=-\infty}^{\infty} g(l+\tilde{\beta}) f(|l+\tilde{\beta}|) \\
& =\int_{0}^{\infty} d u[g(u)+g(-u)] f(u) \\
& \quad+i \int_{0}^{\infty} d u[f(i u)-f(-i u)] \sum_{j= \pm 1} \frac{g(i j u)}{e^{2 \pi(u+i j \tilde{\beta})}-1} .
\end{aligned}
$$

For this case, we can identify

$$
\begin{aligned}
& g(u)=e^{2 \pi i u \Delta z / L} \\
& f(u)=\frac{e^{-i \Delta t \sqrt{\lambda^{2}+p^{2}+\vec{k}^{2}+(2 \pi u / L)^{2}}}}{\sqrt{\lambda^{2}+p^{2}+\vec{k}^{2}+(2 \pi u / L)^{2}}} .
\end{aligned}
$$

Using (2.20), we can write the Wightman function as

$$
W\left(x, x^{\prime}\right)=W_{c s}\left(x, x^{\prime}\right)+W_{c}\left(x, x^{\prime}\right) .
$$

The first term represents the contribution due to the AdS bulk without compactification, which, for our analysis, besides to present dependence on the magnetic fluxes, also depends on the conical structure induced by the presence of the cosmic string. As for the second term, it is induced by the compactification and contains contributions due to the magnetic flux enclosed by the compactified axis. Both expressions are explicitly written in (2.23) and (2.27), respectively. 
The first term on the right-hand side of (2.22), derived from the first integral of (2.20), can be written as

$$
\begin{aligned}
W_{c s}\left(x, x^{\prime}\right)= & \frac{q\left(w w^{\prime}\right)^{\frac{D}{2}} e^{-i e A_{z} \Delta z}}{2(2 \pi)^{D-2} a^{D-1}} \int d \vec{k} e^{i \vec{k} \cdot \Delta \vec{x}_{\|}} \int_{0}^{\infty} d p p J_{\nu}(p w) J_{\nu}\left(p w^{\prime}\right) \\
& \times \sum_{n=-\infty}^{\infty} e^{i n q \Delta \phi} \int_{0}^{\infty} d \lambda \lambda J_{q|n+\alpha|}(\lambda r) J_{q|n+\alpha|}\left(\lambda r^{\prime}\right) \int d k_{z} e^{i k_{z} \Delta z} \frac{e^{-i \Delta t \sqrt{\lambda^{2}+p^{2}+\vec{k}^{2}+k_{z}^{2}}}}{\sqrt{\lambda^{2}+p^{2}+\vec{k}^{2}+k_{z}^{2}}},
\end{aligned}
$$

where we have defined a new variable $k_{z}=2 \pi u / L .{ }^{1}$ Now, performing a Wick rotation and using the identity

$$
\frac{e^{-\Delta \tau \omega}}{\omega}=\frac{2}{\sqrt{\pi}} \int_{0}^{\infty} d s e^{-s^{2} \omega^{2}-\Delta \tau^{2} /\left(4 s^{2}\right)},
$$

the integration over $k_{z}$ can be evaluated, and the result is

$$
\begin{aligned}
W_{c s}\left(x, x^{\prime}\right)= & \frac{q\left(w w^{\prime}\right)^{\frac{D}{2}} e^{-i e A_{z} \Delta z}}{(2 \pi)^{D-2} a^{D-1}} \int d \vec{k} e^{i \vec{k} \cdot \Delta \vec{x}_{\|}} \int_{0}^{\infty} d p p J_{\nu}(p w) J_{\nu}\left(p w^{\prime}\right) \\
& \times \sum_{n=-\infty}^{\infty} e^{i n q \Delta \phi} \int_{0}^{\infty} d \lambda \lambda J_{q|n+\alpha|}(\lambda r) J_{q|n+\alpha|}\left(\lambda r^{\prime}\right) \int_{0}^{\infty} \frac{d s}{s} e^{-s^{2}\left(\lambda^{2}+p^{2}+\vec{k}^{2}\right)-\left(\Delta z^{2}-\Delta t^{2}\right) / 4 s^{2}}
\end{aligned}
$$

Now, let us concentrate on the second term of (2.22). Defining again the variable $k_{z}=2 \pi u / L$, the integral over this variable must be considered in two different intervals; in the first interval $\left[0, \sqrt{\lambda^{2}+p^{2}+\vec{k}^{2}}\right]$, the integral vanishes, so it remains the contribution coming from the second interval, $\left[\sqrt{\lambda^{2}+p^{2}+\vec{k}^{2}}, \infty\right]$. So, taking into account this analysis, we get

$$
\begin{aligned}
W_{c}\left(x, x^{\prime}\right)= & \frac{q\left(w w^{\prime}\right)^{\frac{D}{2}} e^{-i e A_{z} \Delta z}}{(2 \pi)^{D-2} a^{D-1}} \int d \vec{k} e^{i \vec{k} \cdot \Delta \vec{x}_{\|}} \int_{0}^{\infty} d p p J_{\nu}(p w) J_{\nu}\left(p w^{\prime}\right) \\
& \times \sum_{n=-\infty}^{\infty} e^{i n q \Delta \phi} \int_{0}^{\infty} d \lambda \lambda J_{q|n+\alpha|}(\lambda r) J_{q|n+\alpha|}\left(\lambda r^{\prime}\right) \\
& \times \int_{\sqrt{\lambda^{2}+p^{2}+\vec{k}^{2}}}^{\infty} d k_{z} \frac{\cosh \left(\Delta t \sqrt{k_{z}^{2}-\lambda^{2}-p^{2}-\vec{k}^{2}}\right)}{\sqrt{k_{z}^{2}-\lambda^{2}-p^{2}-\vec{k}^{2}}} \sum_{j= \pm 1}^{\infty} \frac{e^{-j k_{z} \Delta z}}{e^{L k_{z}+2 \pi i j \tilde{\beta}}-1} .
\end{aligned}
$$

Developing the series expansion $\left(e^{y}-1\right)^{-1}=\sum_{l=1}^{\infty} e^{-l y}$, and with the help of Ref. [33], it is possible to integrate over $k_{z}$, obtaining

$$
\begin{aligned}
W_{c}\left(x, x^{\prime}\right)= & \frac{q\left(w w^{\prime}\right)^{\frac{D}{2}} e^{-i e A_{z} \Delta z}}{(2 \pi)^{D-2} a^{D-1}} \int d \vec{k} e^{i \vec{k} \cdot \Delta \vec{x}_{\|}} \int_{0}^{\infty} d p p J_{\nu}(p w) J_{\nu}\left(p w^{\prime}\right) \sum_{n=-\infty}^{\infty} e^{i n q \Delta \phi} \int_{0}^{\infty} d \lambda \lambda J_{q|n+\alpha|}(\lambda r) J_{q|n+\alpha|}\left(\lambda r^{\prime}\right) \\
& \left.\times \sum_{j= \pm 1} \sum_{l=1}^{\infty} e^{-2 \pi i \tilde{\beta} j l} K_{0}\left(\sqrt{\left(\lambda^{2}+p^{2}+\vec{k}^{2}\right)\left[(l L+j \Delta z)^{2}-\Delta t^{2}\right.}\right]\right)
\end{aligned}
$$

Clearly, we notice that for $L \rightarrow \infty$ the function above vanishes. By using the integral representation below for the Macdonald function [34],

\footnotetext{
${ }^{1}$ For the case of vanishing magnetic fluxes and the absence of cosmic string, i.e., $q=1$, the expression (2.23) reduces itself to the Wightmann function in an Ads bulk only.
} 


$$
K_{\nu}(x)=\frac{1}{2}\left(\frac{x}{2}\right)^{\nu} \int_{0}^{\infty} d \tau \frac{e^{-\tau-x^{2} / 4 \tau}}{\tau^{\nu+1}}
$$

we can rewrite Eq. (2.27) as

$$
\begin{aligned}
W_{c}\left(x, x^{\prime}\right)= & \frac{q\left(w w^{\prime}\right)^{\frac{D}{2}} e^{-i e A_{z} \Delta z}}{(2 \pi)^{D-2} a^{D-1}} \int d \vec{k} e^{i \vec{k} \cdot \Delta \vec{x}_{\|}} \int_{0}^{\infty} d p p J_{\nu}(p w) J_{\nu}\left(p w^{\prime}\right) \\
& \times \sum_{n=-\infty}^{\infty} e^{i n q \Delta \phi} \int_{0}^{\infty} d \lambda \lambda J_{q|n+\alpha|}(\lambda r) J_{q|n+\alpha|}\left(\lambda r^{\prime}\right) \sum_{j= \pm 1} \sum_{l=1}^{\infty} e^{-2 \pi i \tilde{\beta} j l} \int_{0}^{\infty} \frac{d s}{s} e^{-s^{2}\left(\lambda^{2}+p^{2}+\vec{k}^{2}\right)-\left[(l L+j \Delta z)^{2}-\Delta t^{2}\right] / 4 s^{2}} .
\end{aligned}
$$

Substituting (2.25) and (2.29) into (2.22), and after some manipulations, we get a compact expression for the total Wightman function given below:

$$
\begin{aligned}
W\left(x, x^{\prime}\right)= & \frac{q\left(w w^{\prime}\right)^{\frac{D}{2}} e^{-i e A_{z} \Delta z}}{(2 \pi)^{D-2} a^{D-1}} \int d \vec{k} e^{i \vec{k} \cdot \Delta \vec{x}_{\|}} \int_{0}^{\infty} d p p J_{\nu}(p w) J_{\nu}\left(p w^{\prime}\right) \\
& \times \sum_{n=-\infty}^{\infty} e^{i n q \Delta \phi} \int_{0}^{\infty} d \lambda \lambda J_{q|n+\alpha|}(\lambda r) J_{q|n+\alpha|}\left(\lambda r^{\prime}\right) \sum_{l=-\infty}^{\infty} e^{-2 \pi i \tilde{\beta} l} \int_{0}^{\infty} \frac{d s}{s} e^{-s^{2}\left(\lambda^{2}+p^{2}+\vec{k}^{2}\right)-\left[(l L+j \Delta z)^{2}-\Delta t^{2}\right] / 4 s^{2}}
\end{aligned}
$$

Now, using the integral [34]

$$
\int_{0}^{\infty} d \eta \eta e^{-\eta^{2} s^{2}} J_{\gamma}(\eta \rho) J_{\gamma}\left(\eta \rho^{\prime}\right)=\frac{e^{-\frac{\left(\rho^{2}+\rho^{\prime 2}\right)}{4 s^{2}}}}{2 s^{2}} I_{\gamma}\left(\frac{\rho \rho^{\prime}}{2 s^{2}}\right)
$$

we can integrate over $\lambda, p$, and $\vec{k}$, obtaining

$$
W\left(x, x^{\prime}\right)=\frac{q e^{-i e A_{z} \Delta z}}{2(2 \pi)^{\frac{D}{2}} a^{D-1}}\left(\frac{w w^{\prime}}{r r^{\prime}}\right)^{\frac{D}{2}} \sum_{l=-\infty}^{\infty} e^{-2 \pi i \tilde{\beta} l} \int_{0}^{\infty} d \chi \chi^{\frac{D}{2}-1} e^{-\chi u_{l}^{2} / 2 r r^{\prime}} I_{\nu}\left(\frac{w w^{\prime}}{r r^{\prime}} \chi\right) \sum_{n=-\infty}^{\infty} e^{i q n \Delta \phi} I_{q|n+\alpha|}(\chi),
$$

where we have introduced a new variable, $\chi=r r^{\prime} / 2 s^{2}$, and defined

$$
u_{l}^{2}=r^{2}+r^{\prime 2}+w^{2}+w^{\prime 2}+(l L+\Delta z)^{2}+\Delta \vec{x}_{\|}^{2}-\Delta t^{2} .
$$

The parameter $\alpha$ in Eq. (2.11) can be written in the form

$$
\alpha=n_{0}+\varepsilon, \quad \text { with } \quad|\varepsilon|<\frac{1}{2},
$$

with $n_{0}$ being an integer number. This allow us to sum over the quantum number $n$ in $(2.32)$, using the result obtained in Ref. [6], given as

$$
\begin{aligned}
\sum_{n=-\infty}^{\infty} e^{i q n \Delta \phi} I_{q|n+\alpha|}(\chi)= & \frac{1}{q} \sum_{k} e^{\chi \cos (2 \pi k / q-\Delta \phi)} e^{i \alpha(2 \pi k-q \Delta \phi)} \\
& -\frac{e^{-i q n_{0} \Delta \phi}}{2 \pi i} \sum_{j= \pm 1} j e^{j i \pi q|\varepsilon|} \int_{0}^{\infty} d y \frac{\cosh [q y(1-|\varepsilon|)]-\cosh (|\varepsilon| q y) e^{-i q(\Delta \phi+j \pi)}}{e^{\chi \cosh (y)}[\cosh (q y)-\cos (q(\Delta \phi+j \pi))]}
\end{aligned}
$$

where

$$
-\frac{q}{2}+\frac{\Delta \phi}{\Phi_{0}} \leq k \leq \frac{q}{2}+\frac{\Delta \phi}{\Phi_{0}} .
$$


In short, the obtainment of the above expression is through the integral representation for the modified Bessel function [31],

$$
I_{q|n+\alpha|}(z)=\frac{1}{\pi} \int_{0}^{\pi} d y \cos (q|n+\alpha| y) e^{z \cos y}-\frac{\sin (\pi q|n+\alpha|)}{\pi} \int_{0}^{\infty} d y e^{-z \cosh y-q|n+\alpha| y},
$$

following by the summation of the quantum number $n$ and some additional intermediate steps.

Thus, the substitution of (2.35) into (2.32) allows us to integrate over $\chi$ with the help of [34], yielding

$$
\begin{aligned}
W\left(x, x^{\prime}\right)= & \frac{e^{-i e A_{z} \Delta z}}{(2 \pi)^{\frac{D+1}{2}} a^{D-1}} \sum_{l=-\infty}^{\infty} e^{-2 \pi i \tilde{\beta} l}\left\{\sum_{k} e^{i \alpha(2 \pi k-q \Delta \phi)} F_{\nu-1 / 2}^{(D-1) / 2}\left(u_{l k}\right)\right. \\
& \left.-q \frac{e^{-i q n_{0} \Delta \phi}}{2 \pi i} \sum_{j= \pm 1} j e^{j i \pi q|\varepsilon|} \int_{0}^{\infty} d y \frac{\cosh [(1-|\varepsilon|) q y]-\cosh (|\varepsilon| q y) e^{-i q(\Delta \phi+j \pi)}}{\cosh (q y)-\cos (q(\Delta \phi+j \pi))} F_{\nu-1 / 2}^{(D-1) / 2}\left(u_{l y}\right)\right\}
\end{aligned}
$$

where we have introduced the notation

$$
F_{\gamma}^{\mu}(u)=e^{-i \pi \mu} \frac{Q_{\gamma}^{\mu}(u)}{\left(u^{2}-1\right)^{\mu / 2}}=\frac{\sqrt{\pi} \Gamma(\gamma+\mu+1)}{2^{\gamma+1} \Gamma(\gamma+3 / 2) u^{\gamma+\mu+1}} F\left(\frac{\gamma+\mu}{2}+1, \frac{\gamma+\mu+1}{2} ; \gamma+\frac{3}{2} ; \frac{1}{u^{2}}\right),
$$

with $Q_{\gamma}^{\mu}(u)$ being the associated Legendre function of the second kind and $F(a, b ; c ; z)$ being the hypergeometric function [31]. In (2.38), the arguments of the function $F_{\gamma}^{\mu}$ are given by

$$
\begin{aligned}
& u_{l k}=1+\frac{r^{2}+r^{\prime 2}-2 r r^{\prime} \cos (2 \pi k / q-\Delta \phi)+\Delta w^{2}+(l L+\Delta z)^{2}+\Delta \vec{x}_{\|}^{2}-\Delta t^{2}}{2 w w^{\prime}} \\
& u_{l y}=1+\frac{r^{2}+r^{\prime 2}+2 r r^{\prime} \cosh (y)+\Delta w^{2}+(l L+\Delta z)^{2}+\Delta \vec{x}_{\|}^{2}-\Delta t^{2}}{2 w w^{\prime}} .
\end{aligned}
$$

So, Eq. (2.38) is the most compact expression to the Wightman function. In this format, the $l=0$ component corresponds to the contribution due to the cosmic string only, and $l \neq 0$ is the contribution due to the compactification.

Having obtained the above result, we are in a position to calculate the induced current densities. This new subject is left to the next sections.

\section{BOSONIC CURRENT}

The bosonic current density operator is given by

$$
\begin{aligned}
\hat{j}_{\mu}(x)= & i e\left[\hat{\varphi}^{*}(x) D_{\mu} \hat{\varphi}(x)-\left(D_{\mu} \hat{\varphi}\right)^{*} \hat{\varphi}(x)\right] \\
= & i e\left[\hat{\varphi}^{*}(x) \partial_{\mu} \hat{\varphi}(x)-\hat{\varphi}(x)\left(\partial_{\mu} \hat{\varphi}(x)\right)^{*}\right] \\
& -2 e^{2} A_{\mu}(x)|\hat{\varphi}(x)|^{2} .
\end{aligned}
$$

Its VEV can be evaluated in terms of the positive frequency Wightman function as exhibited below:

$$
\left\langle j_{\mu}(x)\right\rangle=i e \lim _{x^{\prime} \rightarrow x}\left\{\left(\partial_{\mu}-\partial_{\mu}{ }^{\prime}\right) W\left(x, x^{\prime}\right)+2 i e A_{\mu} W\left(x, x^{\prime}\right)\right\} .
$$

As we will see, this VEV is a periodic function of the magnetic fluxes $\Phi_{\phi}$ and $\Phi_{z}$ with period equal to the quantum flux. This can be observed writing the parameter $\alpha$ as in (2.34).

\section{A. Charge density}

Let us begin with the calculation of the charge density. Since $A_{0}=0$, we have

$$
\left\langle j_{0}(x)\right\rangle=i e \lim _{x^{\prime} \rightarrow x}\left(\partial_{t}-\partial_{t}^{\prime}\right) W\left(x, x^{\prime}\right)
$$

Substituting Eq. (2.38) into the above expression, taking the time derivatives and finally the coincidence limit, we obtain a divergent result. To avoid this problem, a regularization procedure is necessary. Many regularization procedures can be applied; however, for the present problem, the most convenient is the Pauli-Villars (PV) gauge-invariant one. Adopting this procedure, regulator fields with large masses are introduced. The number of these fields depends on the specific problem. As we will see below, a single regulator field with mass $M$ is sufficient. By using PV, the regularized VEV of the charge density reads 


$$
\begin{aligned}
\left\langle j^{0}(x)\right\rangle_{\operatorname{Reg}}= & \frac{2 i e}{(2 \pi)^{\frac{D+1}{2}} a^{D+1}} \lim _{t^{\prime} \rightarrow t} \Delta t \sum_{l=-\infty}^{\infty} e^{-2 \pi i \tilde{\beta} l}\left[\sum_{k} e^{2 \pi k i \alpha} \sum_{n=0,1} c_{n} F_{\nu_{(n)}-1 / 2}^{(D+1) / 2}\left(\tilde{u}_{l k}\right)\right. \\
& \left.-\frac{q}{2 \pi i} \sum_{j= \pm 1} j e^{j i \pi q|\varepsilon|} \int_{0}^{\infty} d y \frac{\cosh [(1-|\varepsilon|) q y]-\cosh (|\varepsilon| q y) e^{-i q j \pi}}{\cosh (q y)-\cos (q j \pi)} \sum_{n=0,1} c_{n} F_{\nu_{(n)}-1 / 2}^{(D+1) / 2}\left(\tilde{u}_{l y}\right)\right],
\end{aligned}
$$

where $c_{0}=1 ; \quad \nu_{(0)}=\nu$, given by $(2.11) ; \quad c_{1}=-1$; and $\nu_{(1)}$ is the corresponding parameter associated with the mass $M$. Moreover, in (3.4), the arguments of the functions are

$$
\begin{aligned}
& \tilde{u}_{l k}=1+\frac{4 r^{2} \sin ^{2}(\pi k / q)+(l L)^{2}-\Delta t^{2}}{2 w^{2}} \\
& \tilde{u}_{l y}=1+\frac{4 r^{2} \cosh ^{2}(y)+(l L)^{2}-\Delta t^{2}}{2 w^{2}} .
\end{aligned}
$$

In obtaining the above result, we have used the relation

$$
\partial_{x} F_{\gamma}^{\mu}(u(x))=-\left(\partial_{x} u(x)\right) F_{\gamma}^{\mu+1}(u(x))
$$

by using the recurrence relations for the associated Legendre function of the second kind [31].

We can see from (3.5) that the arguments of the functions $F_{\nu_{(n)}-1 / 2}^{(D+1) / 2}$ above, for $l \neq 0$, are bigger than unity. Consequently, the corresponding compactified contributions inside the brackets of (3.4) are finite, providing a vanishing contribution for the charge density when we take the time coincidence limit, $\Delta t \rightarrow 0$. On the other hand, for cosmic string contribution $(l=0)$ for $k=0$ and for $k \neq 0$ but with $r=0$, the arguments of the functions go to unity for the time coincidence limit. However, in the limit of argument close to 1, by using the asymptotic formula for the hypergeometric function, we get a divergent result below, which does not depend on the parameter $\nu$ :

$$
F_{\nu-1 / 2}^{(D+1) / 2}(u) \approx \frac{\Gamma((D+1) / 2)}{2(u-1)^{(D+1) / 2}} .
$$

So, the divergent behavior of the combination, $F_{\nu_{(0)}-1 / 2}^{(D+1) / 2}(u)-F_{\nu_{1}-1 / 2}^{(D+1) / 2}(u)$, is canceled. Finally, taking the time coincidence limit in (3.4), these contributions also provide a vanishing result. So, we conclude that the charge density vanishes.

Following similar procedure, we also can prove that no radial current density, $\left\langle j^{r}\right\rangle$; currents densities along $w,\left\langle j^{w}\right\rangle$; nor extra dimensions, $\left\langle j^{i}\right\rangle$ for $i=5,6, \ldots$, are induced by this system.

\section{B. Azimuthal current}

The VEV of the azimuthal current density is given by

$$
\left\langle j_{\phi}(x)\right\rangle=i e \lim _{x^{\prime} \rightarrow x}\left\{\left(\partial_{\phi}-\partial_{\phi}^{\prime}\right) W\left(x, x^{\prime}\right)+2 i e A_{\phi} W\left(x, x^{\prime}\right)\right\} .
$$

Substituting (2.19) into the above equation, we can formally express the azimuthal current as

$$
\begin{aligned}
\left\langle j_{\phi}(x)\right\rangle= & -\frac{q e a^{1-D} w^{D}}{(2 \pi)^{D-3} L} \\
& \times \sum_{n=-\infty}^{\infty} q(n+\alpha) \int d \vec{k} \int_{0}^{\infty} \lambda J_{q|n+\alpha|}^{2}(\lambda r) d \lambda \\
& \times \int_{0}^{\infty} p J_{\nu}^{2}(p w) d p \sum_{l=-\infty}^{\infty} \frac{1}{\sqrt{\lambda^{2}+p^{2}+\vec{k}^{2}+\tilde{k}_{l}^{2}}} .
\end{aligned}
$$

Identifying $g(u)=1$ and

$$
f(u)=\frac{1}{\sqrt{\lambda^{2}+p^{2}+\vec{k}^{2}+(2 \pi u / L)^{2}}},
$$

we can use (2.20) to develop the summation on the quantum number $l$. Doing this, the VEV is decomposed as

$$
\left\langle j_{\phi}(x)\right\rangle=\left\langle j_{\phi}(x)\right\rangle_{c s}+\left\langle j_{\phi}(x)\right\rangle_{c}
$$

where $\left\langle j_{\phi}(x)\right\rangle_{c s}$ corresponds the contribution from the cosmic string without compactification, which comes from the first integral on the right-hand side of Eq. (2.20). This component reads

$$
\begin{aligned}
\left\langle j_{\phi}(x)\right\rangle_{c s}= & -\frac{2 q e w^{D}}{(2 \pi)^{D-2} a^{D-1}} \\
& \times \sum_{n=-\infty}^{\infty} q(n+\alpha) \int d \vec{k} \int_{0}^{\infty} d \lambda \lambda J_{q|n+\alpha|}^{2}(\lambda r) \\
& \times \int_{0}^{\infty} p J_{\nu}^{2}(p w) d p \int_{0}^{\infty} \frac{d k_{z}}{\sqrt{\lambda^{2}+p^{2}+\vec{k}^{2}+k_{z}^{2}}},
\end{aligned}
$$

where we have defined $k_{z}=2 \pi u / L$. 
Using the identity

$$
\frac{1}{\sqrt{\lambda^{2}+p^{2}+\vec{k}^{2}+k_{z}^{2}}}=\frac{2}{\sqrt{\pi}} \int_{0}^{\infty} d s e^{-s^{2}\left(\lambda^{2}+p^{2}+\vec{k}^{2}+k_{z}^{2}\right)}
$$

and (2.31), we can perform the integrations over all but the $s$ variable, obtaining

$$
\begin{aligned}
\left\langle j_{\phi}(x)\right\rangle_{c s}= & -\frac{e q^{2} w^{D}}{(2 \pi)^{\frac{D}{2}} a^{D-1} r^{D}} \int_{0}^{\infty} d \chi \chi^{\frac{D}{2}-1} e^{-\chi\left[1+(w / r)^{2}\right]} \\
& \times I_{\nu}\left(\frac{w^{2} \chi}{r^{2}}\right) \sum_{n=-\infty}^{\infty}(n+\varepsilon) I_{q|n+\varepsilon|}(\chi) .
\end{aligned}
$$

We have written $\alpha$ in the form (2.34) and also introduced a new variable, $\chi=r^{2} / 2 s^{2}$. In Ref. [4], a compact expression for the summation over the quantum number $n$ has been derived. We reproduce this result,

$$
\begin{aligned}
& \sum_{n=-\infty}^{\infty}(n+\varepsilon) I_{q|n+\varepsilon|}(\chi) \\
& =\frac{2 \chi}{q^{2}} \sum_{j=1}^{[q / 2]} \sin (2 \pi j / q) \sin (2 \pi j \varepsilon) e^{\chi \cos (2 \pi j / q)} \\
& \quad+\frac{\chi}{q \pi} \int_{0}^{\infty} d y \sinh (y) \frac{e^{-\chi \cosh (y)} g(q, \varepsilon, y)}{\cosh (q y)-\cos (\pi q)},
\end{aligned}
$$

where $[q / 2]$ represents the integer part of $q / 2$ and the prime on the sign of the summation means that in the case $q=2 p$ the term $k=q / 2$ should be taken with the coefficient $1 / 2$. Moreover, the function, $g(q, \varepsilon, y)$, is defined as

$$
\begin{aligned}
g(q, \varepsilon, y)= & \sin (q \pi \varepsilon) \sinh ((1-|\varepsilon|) q y) \\
& -\sinh (q \varepsilon y) \sin ((1-|\varepsilon|) \pi q) .
\end{aligned}
$$

Substituting the above result into (3.14) and with the help of Ref. [34], we get

$$
\begin{aligned}
\left\langle j^{\phi}(x)\right\rangle_{c s}= & \frac{4 e a^{-(1+D)}}{(2 \pi)^{\frac{D+1}{2}}} \\
& \times\left[\sum_{j=1}^{[q / 2]} \sin (2 \pi j / q) \sin (2 \pi j \varepsilon) F_{\nu-1 / 2}^{(D+1) / 2}\left(u_{j}\right)\right. \\
& \left.+\frac{q}{\pi} \int_{0}^{\infty} d y \frac{\sinh (2 y) g(q, \varepsilon, 2 y)}{\cosh (2 q y)-\cos (\pi q)} F_{\nu-1 / 2}^{(D+1) / 2}\left(u_{y}\right)\right],
\end{aligned}
$$

where the arguments of the functions are given by

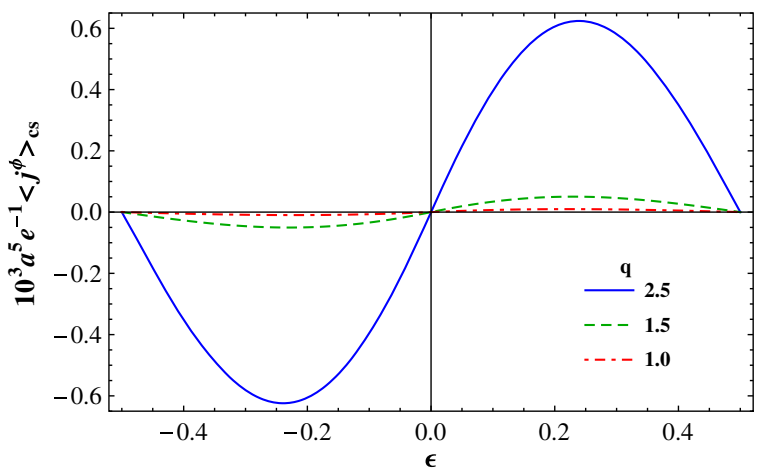

FIG. 1. The azimuthal current density without compactification for $D=4$ in Eq. (3.17) is plotted, in units of $e a^{-5}$, in terms of $\varepsilon$, for $r / w=1 ; m a=1 ; \xi=0$; and $q=1,1.5,2.5$.

$$
\begin{aligned}
& u_{j}=1+2(r / w)^{2} \sin ^{2}(\pi j / q), \\
& u_{y}=1+2(r / w)^{2} \cosh ^{2}(y) .
\end{aligned}
$$

From (3.17), we can see that $\left\langle j^{\phi}(x)\right\rangle_{c s}$ is an odd function of $\varepsilon$ with period equal to quantum flux, $\Phi_{0}=2 \pi / e$; moreover, for $1 \leq q<2$, the first term on the right-hand side is absent. In Fig. 1, we exhibit the behavior of the azimuthal current as a function of $\varepsilon$ for different values of $q$, considering $D=4, \xi=0$ and $r / w=m a=1$. As we can see, the intensity of the current depends strongly on the value of $q$. Increasing $q$, its intensity also increases.

In Fig. 2, we plot the behavior of the azimuthal current, $\left\langle j^{\phi}(x)\right\rangle_{c s}$, as function of $r / w$ for $D=4$, considering $\varepsilon=$ 0.25 and $\xi=0$, for different values of the parameter $q$. In the left panel, we adopted $m a=1$, and in the right panel, $m a=5$.

After the numerical analyses for $\left\langle j^{\phi}(x)\right\rangle_{c s}$, we will develop its behavior for some specific regimes of the physical variables. We start by considering $r / w \rightarrow 0$. We can use the asymptotic formula for the hypergeometric function for small arguments [31] to rewrite Eq. (3.17) as

$$
\begin{aligned}
\left\langle j^{\phi}(x)\right\rangle_{c s} \approx & \frac{4 e \Gamma\left(\frac{D+1}{2}\right)}{(4 \pi)^{\frac{D+1}{2}}}\left(\frac{w}{a r}\right)^{D+1}\left[\sum_{j=1}^{[q / 2]} \frac{\cot (\pi j / q) \sin (2 \pi j \varepsilon)}{\sin ^{D-1}(\pi j / q)}\right. \\
& \left.+\frac{q}{\pi} \int_{0}^{\infty} d y \frac{\tanh (y)}{\cosh ^{D-1}(y)} \frac{g(q, \varepsilon, 2 y)}{\cosh (2 q y)-\cos (\pi q)}\right] .
\end{aligned}
$$

Apart form the conformal factor, $(w / a)^{(D+1)}$, the above expression coincides with the corresponding one in Minkowski background for points near the string [4]. On the other hand, for $r / w \gg 1$, we have 

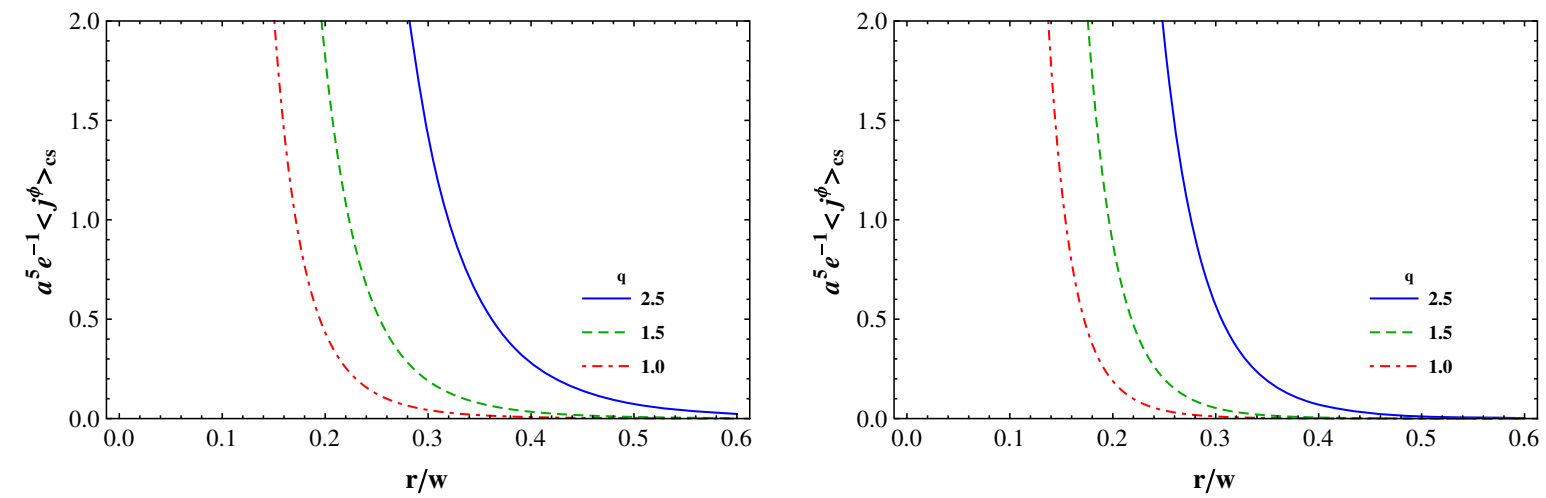

FIG. 2. The azimuthal current density without compactification for $D=4$ in Eq. (3.17) is plotted, in units of $e a^{-5}$, in terms of the proper distance, $r / w$, for $\varepsilon=0.25 ; \xi=0$; and $q=1,1.5,2.5$. The plot on the left is for $m a=1$, while the plot on the right is for $m a=5$.

$$
\begin{aligned}
\left\langle j^{\phi}(x)\right\rangle_{c s} \approx & \frac{2^{1-2 \nu} e \Gamma(D / 2+\nu+1)}{(4 \pi)^{\frac{D}{2}} \Gamma(\nu+1) a^{D+1}}\left(\frac{w}{r}\right)^{D+2 \nu+2} \\
& \times\left\{\sum_{j=1}^{[q / 2]} \frac{\cot (\pi j / q) \sin (2 \pi j \varepsilon)}{\sin ^{D+2 \nu}(\pi j / q)}+\frac{q}{\pi} \int_{0}^{\infty} d y \frac{\tanh (y)}{\cosh ^{D+2 \nu}(y)} \frac{g(q, \varepsilon, 2 y)}{\cosh (2 q y)-\cos (\pi q)}\right\} .
\end{aligned}
$$

Another interesting asymptotic behavior is for $\nu \gg 1$. For this case, Eq. (3.17) reads

$$
\begin{aligned}
\left\langle j^{\phi}(x)\right\rangle_{c s} \approx & \frac{2 e \nu^{D / 2}}{(2 \pi)^{\frac{D}{2}} a^{1+D}}\left[\sum_{j=1}^{[q / 2]} \sin (2 \pi j / q) \sin (2 \pi j \varepsilon) \frac{\left(u_{j}+\sqrt{u_{j}^{2}-1}\right)^{-\nu}}{\left(u_{j}^{2}-1\right)^{\frac{D+2}{4}}}\right. \\
& \left.+\frac{q}{\pi} \int_{0}^{\infty} d y \frac{\sinh (2 y) g(q, \varepsilon, 2 y)}{\cosh (2 q y)-\cos (\pi q)} \frac{\left(u_{y}+\sqrt{u_{y}^{2}-1}\right)^{-\nu}}{\left(u_{y}^{2}-1\right)^{\frac{D+2}{4}}}\right] .
\end{aligned}
$$

For a conformally coupled massless scalar field, we have $\nu=1 / 2$, and by expressing the associated Legendre function in terms of the hypergeometric function [31,34], we can write a more convenient expression for $F_{\nu-1 / 2}^{(D+1) / 2}(u)[5]$, given by

$$
F_{0}^{(D+1) / 2}(u)=-\frac{\Gamma\left(\frac{D+1}{2}\right)}{2}\left[(1+u)^{-(D+1) / 2}-(u-1)^{-(D+1) / 2}\right]
$$

Substituting (3.22) into (3.17), we obtain

$$
\begin{aligned}
\left\langle j^{\phi}(x)\right\rangle_{c s}= & \left(\frac{w}{a}\right)^{D+1}\left\{\frac{4 e \Gamma\left(\frac{D+1}{2}\right)}{(4 \pi)^{\frac{D+1}{2}} r^{(D+1)}}\left[\sum_{j=1}^{[q / 2]} \frac{\cos (\pi j / q) \sin (2 \pi j \varepsilon)}{\sin ^{D}(\pi j / q)}+\frac{q}{\pi} \int_{0}^{\infty} d y \frac{\sinh (y)}{\cosh (2 q y)-\cos (\pi q)} \frac{g(q, \varepsilon, 2 y)}{\cosh ^{D}(y)}\right]\right. \\
& -\frac{2 e \Gamma\left(\frac{D+1}{2}\right)}{(4 \pi)^{\frac{D+1}{2}} r^{(D+1)}}\left[\sum_{j=1}^{\prime} \sin (2 \pi j / q) \sin (2 \pi j \varepsilon)\left(\frac{w^{2}}{r^{2}}+\sin ^{2}(\pi j / q)\right)^{-\frac{D+1}{2}}\right. \\
& \left.\left.+\frac{q}{\pi} \int_{0}^{\infty} d y \frac{\sinh (2 y) g(q, \varepsilon, 2 y)}{\cosh (2 q y)-\cos (\pi q)}\left(\frac{w^{2}}{r^{2}}+\cosh ^{2}(y)\right)^{-\frac{D+1}{2}}\right]\right\} .
\end{aligned}
$$


We notice that two different sets of contributions appear in the expression above. Apart from the conformal factor, the first set coincides with the induced massless scalar azimuthal current in the Minkowski background [4]. It is divergent for $r \rightarrow 0$. As for the second set, it is a new contribution. This part is induced by the boundary located at $w=0$. It is finite at the string's core for $w \neq 0$. In addition, for $r \gg w$, this part tends to cancel the first one. Finally, taking $D=4$ in the above expression, we obtain

$$
\begin{aligned}
\left\langle j^{\phi}(x)\right\rangle_{c s}= & \left(\frac{w}{a}\right)^{5}\left\{\frac{3 e}{32 \pi^{2} r^{5}}\left[\sum_{j=1}^{[q / 2]} \frac{\cot (\pi j / q) \sin (2 \pi j \varepsilon)}{\sin ^{3}(\pi j / q)}+\frac{q}{\pi} \int_{0}^{\infty} d z \frac{\tanh (z)}{\cosh (2 q z)-\cos (\pi q)} \frac{g(q, \epsilon, 2 z)}{\cosh ^{3}(z)}\right]\right. \\
& -\frac{3 e}{64 \pi^{2} r^{5}}\left[\sum_{j=1}^{[q / 2]} \sin (2 \pi j / q) \sin (2 \pi j \varepsilon)\left(\frac{w^{2}}{r^{2}}+\sin ^{2}(\pi j / q)\right)^{-5 / 2}\right. \\
& \left.\left.+\frac{q}{\pi} \int_{0}^{\infty} d y \frac{\sinh (2 y) g(q, \varepsilon, 2 y)}{\cosh (2 q y)-\cos (\pi q)}\left(\frac{w^{2}}{r^{2}}+\cosh ^{2}(y)\right)^{-5 / 2}\right]\right\}
\end{aligned}
$$

The compactified contribution for the azimuthal current, $\left\langle j_{\phi}(x)\right\rangle_{c}$, can be obtained using (2.26). So, we have

$$
\begin{aligned}
\left\langle j_{\phi}(x)\right\rangle_{c}= & -\frac{2 q e w^{D}}{(2 \pi)^{D-2} a^{D-1}} \sum_{n=-\infty}^{\infty} q(n+\alpha) \int d \vec{k} \int_{0}^{\infty} \lambda J_{q|n+\alpha|}^{2}(\lambda r) d \lambda \\
& \times \int_{0}^{\infty} p J_{\nu}^{2}(p w) d p \int_{\sqrt{\lambda^{2}+p^{2}+\vec{k}^{2}}}^{\infty} \frac{d k_{z}}{\sqrt{k_{z}^{2}-\lambda^{2}-p^{2}-\vec{k}^{2}}} \sum_{j= \pm 1} \frac{1}{e^{L k_{z}+2 \pi i j \tilde{\beta}}-1} .
\end{aligned}
$$

To proceed with our analysis, it is necessary to use the series expansion $\left(e^{y}-1\right)^{-1}=\sum_{l=1}^{\infty} e^{-l y}$, and with the help of Ref. [34], we can integrate over $k_{z}$, obtaining

$$
\begin{aligned}
\left\langle j_{\phi}(x)\right\rangle_{c}= & -\frac{4 q e w^{D}}{(2 \pi)^{D-2} a^{D-1}} \sum_{l=1}^{\infty} \cos (2 \pi i \tilde{\beta}) \sum_{n=-\infty}^{\infty} q(n+\alpha) \int d \vec{k} \int_{0}^{\infty} d \lambda \lambda J_{q|n+\alpha|}^{2}(\lambda r) \\
& \times \int_{0}^{\infty} d p p J_{\nu}^{2}(p w) K_{0}\left(l L \sqrt{\lambda^{2}+p^{2}+\vec{k}^{2}}\right)
\end{aligned}
$$

Using the integral representation for the Macdonald function given in (2.28), it is possible to integrate over the variables $\lambda$, $p$, and $\vec{k}$, getting

$$
\begin{aligned}
\left\langle j_{\phi}(x)\right\rangle_{c}= & -\frac{2 q^{2} e w^{D}}{(2 \pi)^{D / 2} a^{D-1} r^{D}} \sum_{l=1}^{\infty} \cos (2 \pi l \tilde{\beta}) \int_{0}^{\infty} d \chi \chi^{(D-2) / 2} e^{-\chi\left[1+\left(l^{2} L^{2}+2 w^{2}\right) / 2 r^{2}\right]} \\
& \times I_{\nu}\left(\frac{w^{2} \chi}{r^{2}}\right) \sum_{n=-\infty}^{\infty}(n+\varepsilon) I_{q|n+\varepsilon|}(\chi)
\end{aligned}
$$

where we have written $\alpha$ in the form of (2.34) and defined the variable, $\chi=\frac{2 t r^{2}}{(l L)^{2}}$. Now, using the sum formula given in (3.15), we are able to integrate over $\chi$, obtaining the final form of the contribution to the azimuthal current density induced by the compactification,

$$
\begin{aligned}
\left\langle j^{\phi}(x)\right\rangle_{c}= & \frac{8 e a^{-(D+1)}}{(2 \pi)^{(D+1) / 2}} \sum_{l=1}^{\infty} \cos (2 \pi l \tilde{\beta})\left[\sum_{j=1}^{[q / 2]} \sin (2 \pi j / q) \sin (2 \pi j \varepsilon) F_{\nu-1 / 2}^{(D+1) / 2}\left(v_{l j}\right)\right. \\
& \left.+\frac{q}{\pi} \int_{0}^{\infty} d y \frac{\sinh (2 y) g(q, \varepsilon, 2 y)}{\cosh (2 q y)-\cos (\pi q)} F_{\nu-1 / 2}^{(D+1) / 2}\left(v_{l y}\right)\right],
\end{aligned}
$$


with

$$
\begin{aligned}
& v_{l j}=1+\frac{(l L)^{2}+4 r^{2} \sin ^{2}(\pi j / q)}{2 w^{2}} \\
& v_{l y}=1+\frac{(l L)^{2}+4 r^{2} \cosh ^{2}(y)}{2 w^{2}} .
\end{aligned}
$$

From the above expression, we can see that the contribution due to the compactification on the azimuthal current density is an even function of the parameter $\tilde{\beta}$ and is an odd function of the magnetic flux along the core of the string, with period equal to $\Phi_{0}$. In particular, in the case of an untwisted bosonic field, $\left\langle j^{\phi}(x)\right\rangle_{\mathrm{c}}$ is an even function of the magnetic flux enclosed by the compactified dimension. In Fig. 3, we plot (3.28) as function of $\tilde{\beta}$ for $D=4$, considering $m a=1, \xi=0, \varepsilon=0.25$, and different values of $q$. As we can see, besides $\left\langle j^{\phi}(x)\right\rangle_{c}$ to present a strong dependence on the parameter $q$, its direction depends on the value of $\tilde{\beta}$.

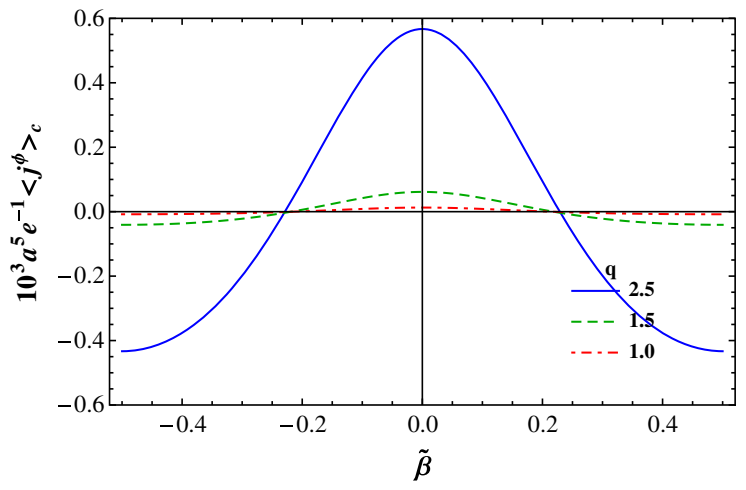

FIG. 3. The azimuthal current density induced by compactification for $D=4$ is plotted, in units of $e a^{-5}$, in terms of $\tilde{\beta}$ for $m a=1 ; \xi=0 ; \varepsilon=0.25$, and $q=1,1.5,2.5$.

In the regime $L / w \gg 1$, Eq. (3.28) presents the following asymptotic behavior:

$$
\begin{aligned}
\left\langle j^{\phi}(x)\right\rangle_{c} \approx & \frac{2^{1-2 \nu} e \Gamma(D / 2+\nu+1)}{(4 \pi)^{\frac{D}{2}} \Gamma(\nu+1) a^{D+1}}\left(\frac{w}{L}\right)^{D+2 \nu+2} \sum_{l=1}^{\infty} \cos (2 \pi i \tilde{\beta}) \\
& \times\left\{\sum_{j=1}^{[q / 2]} \sin (2 \pi j / q) \sin (2 \pi j \varepsilon)\left[\frac{l^{2}}{4}+\left(\frac{r}{L}\right)^{2} \sin ^{2}(\pi j / q)\right]^{-\frac{D}{2}-\nu-1}\right. \\
& \left.+\frac{q}{\pi} \int_{0}^{\infty} d y \frac{\sinh (2 y) g(q, \varepsilon, 2 y)}{\cosh (2 q y)-\cos (\pi q)}\left[\frac{l^{2}}{4}+\left(\frac{r}{L}\right)^{2} \cosh ^{2}(y)\right]^{-\frac{D}{2}-\nu-1}\right\} .
\end{aligned}
$$

For a conformally coupled massless scalar field and taking $D=4$, a much simpler expression can be provided. It reads

$$
\begin{aligned}
\left\langle j^{\phi}(x)\right\rangle_{c}= & \left(\frac{w}{a L}\right)^{5} \frac{3 e}{\pi^{2}}\left\{\sum_{j=1}^{[q / 2]} \sin (2 \pi j / q) \sin (2 \pi j \varepsilon)\left[G_{c}\left(\tilde{\beta}, \rho_{j}\right)-G_{c}\left(\tilde{\beta}, \sigma_{j}\right)\right]\right. \\
& \left.+\frac{q}{\pi} \int_{0}^{\infty} d y \frac{\sinh (2 y) g(q, \varepsilon, 2 y)}{\cosh (2 q y)-\cos (\pi q)}\left[G_{c}(\tilde{\beta}, \eta(y))-G_{c}(\tilde{\beta}, \tau(y))\right]\right\},
\end{aligned}
$$

where we have defined the function

$$
G_{c}(\tilde{\beta}, x)=\sum_{l=1}^{\infty} \frac{\cos (2 \pi l \tilde{\beta})}{\left(l^{2}+x^{2}\right)^{5 / 2}}
$$

and introduced new variables

$$
\begin{aligned}
& \rho_{j}=\frac{2 r \sin (\pi j / q)}{L}, \quad \eta(y)=\frac{2 r \cosh (y)}{L} \\
& \sigma_{j}=\frac{2}{L} \sqrt{w^{2}+r^{2} \sin ^{2}(\pi j / q)}, \quad \tau(y)=\frac{2}{L} \sqrt{w^{2}+r^{2} \cosh ^{2}(y)} .
\end{aligned}
$$

Similarly to what happened with (3.23), two different contributions appear in (3.31). The positive contribution is due to the compactification only, and the negative one is induced by the boundary located at $w=0$. Also, we can observe that for $r \gg w$ the latter tends to cancel the former. 


\section{Current along the compactified dimension}

In this section, we want to analyze the current density along the compactified axis, named the axial current. As we shall see, due to the compactification, an axial current will be induced. This current goes to zero in the limit $L \rightarrow \infty$. The VEV of the axial current is calculated by

$$
\left\langle j_{z}(x)\right\rangle=i e \lim _{x^{\prime} \rightarrow x}\left\{\left(\partial_{z}-\partial_{z}^{\prime}\right) W\left(x, x^{\prime}\right)+2 i e A_{z} W\left(x, x^{\prime}\right)\right\} .
$$

Substituting Eq. (2.19) into the above expression and using the fact that $A_{z}=-\Phi_{z} / L$, we obtain

$$
\begin{aligned}
\left\langle j_{z}(x)\right\rangle= & -\frac{q e a^{1-D} w^{D}}{(2 \pi)^{D-3} L} \sum_{n=-\infty}^{\infty} \int d \vec{k} \int_{0}^{\infty} \lambda J_{q|n+\alpha|}^{2}(\lambda r) d \lambda \\
& \times \int_{0}^{\infty} p J_{\nu}^{2}(p w) d p \sum_{l=-\infty}^{\infty} \frac{\tilde{k}_{l}}{\sqrt{\lambda^{2}+p^{2}+\vec{k}^{2}+\tilde{k}_{l}^{2}}}
\end{aligned}
$$

where $\tilde{k}_{l}$ is given by (2.14).

The sum over the quantum number $l$ is again evaluated by using the Abel-Plana formula given in (2.20). In this case, we identify $g(u)=2 \pi u / L$, and $f(u)$ is given by (3.10). The first integral on the right-hand side is zero due the fact that $g(u)$ is an odd function. Therefore, it remains only the second integral. It reads

$$
\begin{aligned}
\left\langle j_{z}(x)\right\rangle= & -\frac{2 i_{q e a}{ }^{1-D} w^{D}}{(2 \pi)^{D-2}} \int d \vec{k} \int_{0}^{\infty} p J_{\nu}^{2}(p w) d p \\
& \times \sum_{n=-\infty}^{\infty} \int_{0}^{\infty} \lambda J_{q|n+\alpha|}^{2}(\lambda r) d \lambda \int_{\sqrt{\lambda^{2}+p^{2}+\vec{k}^{2}}}^{\infty} d k_{z} \\
& \times \frac{k_{z}}{\sqrt{k_{z}^{2}-\lambda^{2}-p^{2}-\vec{k}^{2}}} \sum_{j= \pm 1} \frac{j}{e^{L k_{z}+2 \pi i j \tilde{\beta}}-1},
\end{aligned}
$$

where we have defined the variable $k_{z}=2 \pi u / L$. Again, by using the series expansion, $\left(e^{y}-1\right)=\sum_{l=1}^{\infty} e^{-l y}$, in the above expression, we have

$$
\begin{aligned}
\left\langle j_{z}(x)\right\rangle= & -\frac{4 q e a^{1-D} w^{D}}{(2 \pi)^{D-2}} \sum_{l=1}^{\infty} \sin (2 \pi l \tilde{\beta}) \\
& \times \int d \vec{k} \int_{0}^{\infty} p J_{\nu}^{2}(p w) d p \sum_{n=-\infty}^{\infty} \int_{0}^{\infty} \lambda J_{q|n+\alpha|}^{2}(\lambda r) d \lambda \\
& \times \int_{\sqrt{\lambda^{2}+p^{2}+\vec{k}^{2}}}^{\infty} d k_{z} \frac{k_{z} e^{-l L k_{z}}}{\sqrt{k_{z}^{2}-\lambda^{2}-p^{2}-\vec{k}^{2}}}
\end{aligned}
$$

We can evaluate the integral over $k_{z}$ with the help of Ref. [34], and the result is given terms of the Macdonald function of the first order, $K_{1}(z)$. Using the integral representation (2.28) again and the fact that $K_{\nu}(y)=$ $K_{-\nu}(y)$, we obtain

$$
\begin{gathered}
\int_{\sqrt{\lambda^{2}+p^{2}+\vec{k}^{2}}}^{\infty} d k_{z} \frac{k_{z} e^{-l L k_{z}}}{\sqrt{k_{z}^{2}-\lambda^{2}-p^{2}-\vec{k}^{2}}} \\
=\frac{1}{l L} \int_{0}^{\infty} d t e^{-t-(l L)^{2}\left(\lambda^{2}+p^{2}+\vec{k}^{2}\right) / 4 t} .
\end{gathered}
$$

Substituting (3.38) into (3.37), it is possible to evaluate the integrals over $\lambda, p$, and $\vec{k}$, obtaining

$$
\begin{aligned}
\left\langle j_{z}(x)\right\rangle= & -\frac{2 q e a^{1-D} w^{D} L}{(2 \pi)^{D / 2} r^{D+2}} \sum_{l=1}^{\infty} l \sin (2 \pi l \tilde{\beta}) \\
& \times \int_{0}^{\infty} d \chi \chi^{D / 2} e^{-\chi\left[1+\left(l^{2} L^{2}+2 w^{2}\right) / 2 r^{2}\right]} \\
& \times I_{\nu}\left(\frac{w^{2} \chi}{r^{2}}\right) \sum_{n=-\infty}^{\infty} I_{q|n+\varepsilon|}(\chi)
\end{aligned}
$$

where we have introduced the variable $\chi=2 t r^{2} /(l L)^{2}$. The summation over $n$ can be found in Ref. [4] and also given by (2.35) by taking $\Delta \varphi=0$. This sum is

$$
\begin{aligned}
\sum_{n=-\infty}^{\infty} I_{q|n+\varepsilon|}(\chi)= & \frac{e^{\chi}}{q}-\frac{1}{\pi} \int_{0}^{\infty} d y \frac{e^{-\chi \cosh (y)} f(q, \varepsilon, y)}{\cosh (q y)-\cos (\pi q)} \\
& +\frac{2}{q} \sum_{k=1}^{[q / 2]} \cos (2 \pi k \varepsilon) e^{\chi \cos (2 \pi k / q)}
\end{aligned}
$$

The function, $f(q, \varepsilon, y)$, is defined as

$$
\begin{aligned}
f(q, \varepsilon, y)= & \sin [(1-|\varepsilon|) q \pi] \cosh (|\varepsilon| q y) \\
& +\sin (|\varepsilon| q \pi) \cosh [(1-|\varepsilon|) q y] .
\end{aligned}
$$

Finally substituting (3.40) into (3.39), we get

$$
\begin{aligned}
\left\langle j_{z}(x)\right\rangle= & -\frac{2 q e a^{1-D} w^{D} L}{(2 \pi)^{D / 2} r^{D+2}} \sum_{l=1}^{\infty} l \sin (2 \pi l \tilde{\beta}) \\
& \times \int_{0}^{\infty} d \chi \chi^{D / 2} e^{-\chi\left[1+\left(l^{2} L^{2}+2 w^{2}\right) / 2 r^{2}\right]} I_{\nu}\left(\frac{w^{2} \chi}{r^{2}}\right) \\
& \times\left[\frac{e^{\chi}}{q}-\frac{1}{\pi} \int_{0}^{\infty} d y \frac{e^{-\chi \cosh (y)} f(q, \varepsilon, y)}{\cosh (q y)-\cos (\pi q)}\right. \\
& \left.+\frac{2}{q} \sum_{k=1}^{[q / 2]} \cos (2 \pi k \varepsilon) e^{\chi \cos (2 \pi k / q)}\right] .
\end{aligned}
$$

At this point, we may decompose the current above as

$$
\left\langle j_{z}(x)\right\rangle=\left\langle j_{z}(x)\right\rangle_{c}^{(0)}+\left\langle j_{z}(x)\right\rangle_{c}^{(q, \varepsilon)}
$$


where the first term on the right-hand side of the above expression,

$$
\left\langle j^{z}(x)\right\rangle_{c}^{(0)}=\frac{4 e a^{-(1+D)} L}{(2 \pi)^{\frac{D+1}{2}}} \sum_{l=1}^{\infty} l \sin (2 \pi l \tilde{\beta}) F_{\nu-1 / 2}^{(D+1) / 2}\left(u_{l 0}\right),
$$

with $u_{l 0}$ given below in (3.47), is purely due to the compactification. It does not depend on $\varepsilon$ and $q$. For a conformally coupled massless scalar field and taking $D=4$, this contribution reads

$$
\begin{aligned}
\left\langle j^{z}(x)\right\rangle_{c}^{(0)}= & \left(\frac{w}{a}\right)^{5} \frac{3 e}{2 \pi^{2} L^{4}} \\
& \times\left\{\sum_{l=1}^{\infty} \frac{\sin (2 \pi \tilde{\beta} l)}{l^{4}}-\sum_{l=1}^{\infty} \frac{l \sin (2 \pi \tilde{\beta} l)}{\left[l^{2}+\left(\frac{2 w}{L}\right)^{2}\right]^{\frac{5}{2}}}\right\} .
\end{aligned}
$$

The second contribution to the axial current depends on the magnetic fluxes and the parameter associated with the cosmic string. It is given by

$$
\begin{aligned}
\left\langle j^{z}(x)\right\rangle_{c}^{(q, \varepsilon)}= & \frac{8 e a^{-(1+D)} L}{(2 \pi)^{\frac{D+1}{2}}} \sum_{l=1}^{\infty} l \sin (2 \pi l \tilde{\beta}) \\
& \times\left[\sum_{k=1}^{[q / 2]} \cos (2 \pi k \varepsilon) F_{\nu-1 / 2}^{(D+1) / 2}\left(u_{l k}\right)\right. \\
& \left.-\frac{q}{\pi} \int_{0}^{\infty} d y \frac{f(q, \varepsilon, 2 y)}{\cosh (2 q y)-\cos (\pi q)} F_{\nu-1 / 2}^{(D+1) / 2}\left(u_{l y}\right)\right],
\end{aligned}
$$

where we have adopted the following notation:

$$
\begin{aligned}
& u_{l k}=1+\frac{(l L)^{2}+4 r^{2} \sin ^{2}(\pi k / q)}{2 w^{2}} \\
& u_{l y}=1+\frac{(l L)^{2}+4 r^{2} \cosh ^{2}(y)}{2 w^{2}}
\end{aligned}
$$

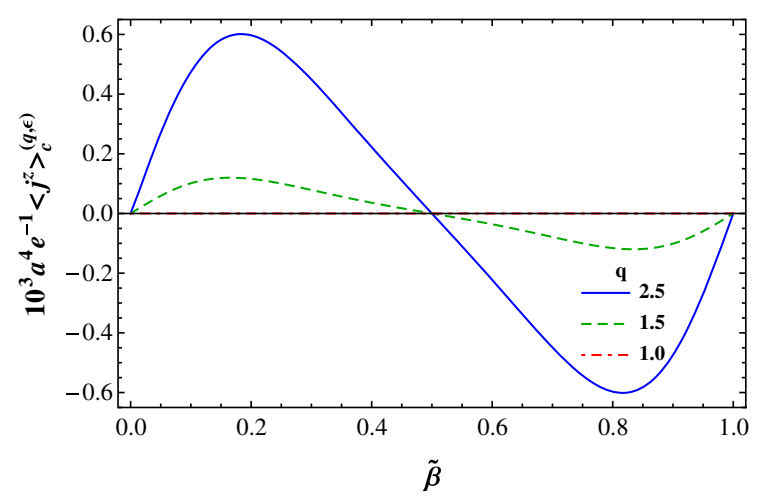

Notice that this term depends on the radial distance, $r$, and is finite on the string's core. We also can notice that the axial current vanishes for integer and half-integer values of $\tilde{\beta}$. In Fig. 4, we plot the axial current density, Eq. (3.46), for $D=4$ as function of $\tilde{\beta}$ for $\varepsilon=0$ and $\varepsilon=0.25$, considering $m a=1, \xi=0, L / a=1$, and different values of $q$.

We can also analyze the axial current in the regime $L / w \gg 1$. Using the asymptotic behavior for the hypergeometric function for large arguments, we get the following expression:

$$
\begin{aligned}
\left\langle j^{z}(x)\right\rangle_{c}^{(q, \varepsilon)} \approx & \frac{2^{1-2 \nu} e \Gamma(D / 2+\nu+1) L}{(4 \pi)^{\frac{D}{2}} \Gamma(\nu+1) a^{D+1}}\left(\frac{w}{L}\right)^{D+2 \nu+2} \\
& \times \sum_{l=1}^{\infty} l \sin (2 \pi l \tilde{\beta}) \\
& \times\left\{\sum_{k=1}^{[q / 2]} \cos (2 \pi k \varepsilon)\left[\frac{l^{2}}{4}+\left(\frac{r}{L}\right)^{2} \sin ^{2}(\pi k / q)\right]^{-\frac{D}{2}-\nu-1}\right. \\
& -\frac{q}{\pi} \int_{0}^{\infty} d y \frac{f(q, \varepsilon, 2 y)}{\cosh (2 q y)-\cos (\pi q)} \\
& \left.\times\left[\frac{l^{2}}{4}+\left(\frac{r}{L}\right)^{2} \cosh ^{2}(y)\right]^{-\frac{D}{2}-\nu-1}\right\}
\end{aligned}
$$

Finally, for a conformally coupled massless scalar field and assuming $D=4$, we obtain

$$
\begin{aligned}
\left\langle j^{z}(x)\right\rangle_{c}^{(q, \varepsilon)}= & \left(\frac{w}{a}\right)^{5} \frac{3 e}{\pi^{2} L^{4}} \\
& \times\left\{\sum_{k=1}^{[q / 2]} \cos (2 \pi k \varepsilon)\left[V_{c}\left(\tilde{\beta}, \rho_{k}\right)-V_{c}\left(\tilde{\beta}, \sigma_{k}\right)\right]\right. \\
& -\frac{q}{\pi} \int_{0}^{\infty} d y \frac{f(q, \varepsilon, 2 y)}{\cosh (2 q y)-\cos (\pi q)} \\
& \left.\times\left[V_{c}(\tilde{\beta}, \eta(y))-V_{c}(\tilde{\beta}, \tau(y))\right]\right\}
\end{aligned}
$$

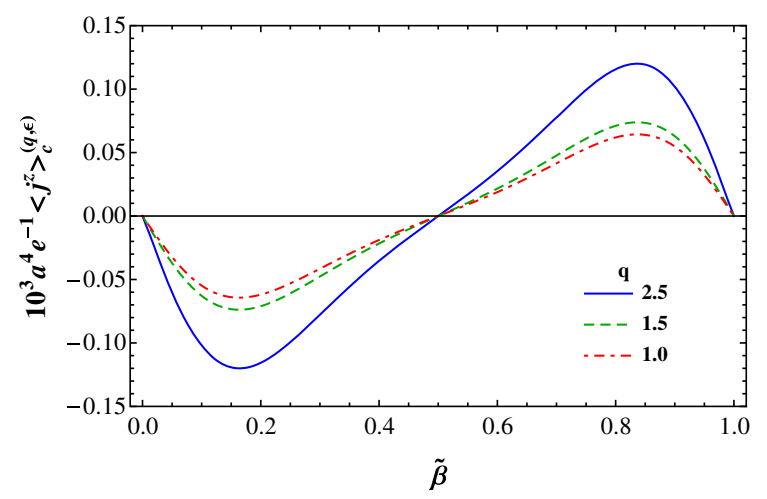

FIG. 4. The axial current density is plotted for $D=4$, in units of $e a^{-4}$, as a function of $\tilde{\beta}$ for $m a=1 ; \xi=0 ; L / a=1$; and $q=1,1.5$, 2.5. In the left plot, we consider $\varepsilon=0$, while in the right plot, we take $\varepsilon=0.25$. 
where we have introduced the function

$$
V_{c}(\tilde{\beta}, x)=\sum_{l=1}^{\infty} \frac{l \sin (2 \pi \tilde{\beta} l)}{\left(l^{2}+x^{2}\right)^{5 / 2}}
$$

in the integrands of (3.49), with the corresponding arguments defined in (3.33).

\section{CONCLUSIONS}

In this paper, we have investigated the induced scalar current density, $\left\langle j^{\mu}\right\rangle$, in a $(D+1)$-dimensional AdS space, with $D \geq 4$, admitting the presence of a cosmic string having a magnetic flux running along its axis. In addition, we assume the compactification of just one extra dimension in a circle of perimeter $L$ and the existence of a constant vector potential along this direction. This compactification is implemented by assuming that the matter field obeys a quasiperiodicity condition along it, Eq. (2.6). To develop this analysis, we construct the positive energy Wightman function, by solving the Klein-Gordon equation in the corresponding background. By using the Poincaré coordinate and admitting a general curvature coupling constant, the normalized solution is given by (2.17). The Wightman function is evaluated by summing over all set of normalized solution (2.19). By using the Abel-Plana summation formula, Eq. (2.20), the Wightman function is decomposed in two contributions, one due only to the cosmic string in the AdS background and the other induced by the compactification. Fortunately, we were able to express this function in a compact form in Eq. (2.38).

In our analysis, we have proven that only azimuthal and axial current densities are induced. Because of the compactification, the azimuthal current has been decomposed in two parts. The first one corresponds to the expression in the geometry of a cosmic string in AdS bulk without compactification, and the second is induced by the compactification; both are presented by Eqs. (3.17) and (3.28), respectively. Both contributions are odd functions of $\varepsilon$, with a period equal to the quantum flux $\Phi_{0}$. This is an Aharonov-Bohm-like effect. The pure cosmic string contribution is plotted for $D=4$, in units of the inverse of $a^{5} e^{-1}$, as a function of $\varepsilon$ as shown in Fig. 1. From this graph, we can see that the intensity of this current increases with the parameter $q$; also, we have plotted this contribution for two different values of the product $m a$ as a function of dimensionless variable $r / w$ for different values of $q$. These graphs are presented in Fig. 2. By them, we can see a strong decay in the intensity of $\langle j\rangle_{c s}$; moreover, carefully, we can identify that for a bigger value of $m a$ the decay is more accentuated. Some asymptotic expressions for this current are provided for specific limiting cases of the physical parameter of the model. For small and larger values of $r / w$, the corresponding asymptotic expressions are given by (3.19) and (3.20), respectively. For $\nu \gg 1$, it is given by (3.21). Finally, for a conformally coupled massless field, the induced current assumes the form (3.23).

As to the azimuthal current density induced by the compactification, Eq. (3.28), we can observe that it is an even function of the parameter $\tilde{\beta}$ and is an odd function of the magnetic flux along the core of the string, with a period equal to $\Phi_{0}$. Its dependence on $\tilde{\beta}$ is plotted in Fig. 3, considering the interval $[-0.5,0.5]$ and different values of $q$. Here, we also observe that this component depends strongly on $q$. Its asymptotic behavior for large values of $L / w$ is presented in (3.30), where we can observe that this current decays with a specific power of $w / L$.

Because of the compactification, there appears an induced current along the compactified extra dimension presented in a complete expression by (3.42). It has a purely compactification origin and vanishes when $\tilde{\beta}=0$, $1 / 2$, and 1 . This current can be expressed as the sum of two terms. One of them is given by Eq. (3.44). It is explicitly shown that it is independent of the radial distance $r$, the cosmic string parameter $q$, and $\varepsilon$. The other contribution is given by Eq. (3.46). It depends on the magnetic fluxes and the planar angle deficit, and it is an odd function of the parameter $\tilde{\beta}$ and is an even function of $\varepsilon$, with a period equal to the quantum flux $\Phi_{0}$. For the particular case in which $\beta=0$, Eq. (3.46) becomes an odd function of the magnetic flux enclosed by the compactified dimension. A plot of the azimuthal current as function $\tilde{\beta}$ is presented in Fig. 4 for two different values of $\varepsilon$ and considering $D=4$. From this graph, we can see that the amplitude of the current increases with the parameter $q$ and the effect of $\varepsilon$ is to change the orientation of the current.

Before concluding, we would like to mention that the currents densities analyzed in this paper refer to the vacuum ones induced by the presence of magnetic fluxes and the compactification. As was exhibited by all the graphs provided, the planar angle deficit associated with the cosmic string spacetime increases the intensity of the azimuthal current density, and the compactification introduces an additional contribution to it; moreover, the latter induces a new current density along the compactified dimension.

\section{ACKNOWLEDGMENTS}

W. O.S. thanks Coordenação de Aperfeiçoamento de Pessoal de Nível Superior (CAPES) for financial support. H.F. M. and E. R. B. M. thank Conselho Nacional de Desenvolvimento Científico e Tecnológico (CNPq) for partial financial support under Grants No. 305379/20178, and No. 313137/2014-5, respectively. 
[1] V. M. Mostepanenko and N. N. Trunov, The Casimir Effect and Its Applications (Clarendon, Oxford, 1997), p. 199.

[2] M. Bordag, G. L. Klimchitskaya, U. Mohideen, and V. M. Mostepanenko, Advances in the Casimir Effect (Oxford University, Oxford, 2009), Vol. 145.

[3] K. A. Milton, The Casimir Effect: Physical Manifestations of Zero-Point Energy (World Scientific, Singapore, 2001), p. 301 .

[4] E. A. F. Bragana, H. F. Santana Mota, and E. R. Bezerra de Mello, Induced vacuum bosonic current by magnetic flux in a higher dimensional compactified cosmic string spacetime, Int. J. Mod. Phys. D 24, 1550055 (2015).

[5] E. R. Bezerra de Mello, A. A. Saharian, and V. Vardanyan, Induced vacuum currents in anti-de Sitter space with toral dimensions, Phys. Lett. B 741, 155 (2015).

[6] E. R. Bezerra de Mello, V. B. Bezerra, A. A. Saharian, and H. H. Harutyunyan, Vacuum currents induced by a magnetic flux around a cosmic string with finite core, Phys. Rev. D 91, 064034 (2015).

[7] E. R. Bezerra de Mello and A. A. Saharian, Fermionic current induced by magnetic flux in compactified cosmic string spacetime, Eur. Phys. J. C 73, 2532 (2013).

[8] E. R. Bezerra de Mello, Induced fermionic current densities by magnetic flux in higher dimensional cosmic string spacetime, Classical Quantum Gravity 27, 095017 (2010).

[9] E. R. Bezerra de Mello, V. B. Bezerra, A. A. Saharian, and V. M. Bardeghyan, Fermionic current densities induced by magnetic flux in a conical space with a circular boundary, Phys. Rev. D 82, 085033 (2010).

[10] A. Mohammadi, E. R. Bezerra de Mello, and A. A. Saharian, Induced fermionic currents in de Sitter spacetime in the presence of a compactified cosmic string, Classical Quantum Gravity 32, 135002 (2015).

[11] M. S. Maior de Sousa, R. F. Ribeiro, and E. R. Bezerra de Mello, Induced fermionic current by a magnetic tube in the cosmic string spacetime, Phys. Rev. D 93, 043545 (2016).

[12] L. M. Sokolowski, The bizarre anti-de Sitter spacetime, Int. J. Geom. Methods Mod. Phys. 13, 1630016 (2016).

[13] E. R. B. de Mello and A. A. Saharian, Vacuum polarization induced by a cosmic string in anti-de Sitter spacetime, J. Phys. A 45, 115402 (2012).

[14] A. de Pdua Santos and E. R. Bezerra de Mello, Non-Abelian cosmic strings in de Sitter and anti-de Sitter space, Phys. Rev. D 94, 063524 (2016).

[15] E. R. Bezerra de Mello, A. A. Saharian, and M. R. Setare, Vacuum densities for a brane intersecting the AdS boundary, Phys. Rev. D 92, 104005 (2015).

[16] S. Bellucci, A. A. Saharian, and V. Vardanyan, Vacuum currents in braneworlds on AdS bulk with compact dimensions, J. High Energy Phys. 11 (2015) 092.

[17] S. Bellucci, A. A. Saharian, and V. Vardanyan, Hadamard function and the vacuum currents in braneworlds with compact dimensions: Two-brane geometry, Phys. Rev. D 93, 084011 (2016).

[18] O. Aharony, S. S. Gubser, J. M. Maldacena, H. Ooguri, and Y. Oz, Large $\mathrm{N}$ field theories, string theory and gravity, Phys. Rep. 323, 183 (2000).
[19] P. Brax and C. van de Bruck, Cosmology and brane worlds: A review, Classical Quantum Gravity 20, R201 (2003).

[20] A. Vilenkin and E. P. S. Shellard, Cosmic Strings and Other Topological Defects, in Cambridge Monographs on Mathematical Physics (Cambridge University Press, Cambridge, England, 1994).

[21] M. Hindmarsh and T. Kibble, Cosmic strings, Rep. Prog. Phys. 58, 477 (1995).

[22] B. Allen and E. P. S. Shellard, On the evolution of cosmic strings, in Proceedings of a Workshop on the Formation and Evolution of Cosmic Strings Supported by the SERC and Held in Cambridge, 1989, edited by G. W. Gibbons, S. W. Hawking, and T. Vachaspati (Cambridge University Press, Cambridge, England, 1990), pp. 421-448.

[23] E. J. Copeland, L. Pogosian, and T. Vachaspati, Seeking string theory in the Cosmos, Classical Quantum Gravity 28, 204009 (2011).

[24] M. Hindmarsh, Signals of inflationary models with cosmic strings, Prog. Theor. Phys. Suppl. 190, 197 (2011).

[25] D. F. Chernoff and S.H. Henry Tye, Inflation, string theory and cosmic strings, One Hundred Years of General Relativity: From Genesis and Empirical Foundations to Gravitational Waves, Cosmology and Quantum Gravity (World Scientific, Singapore, 2017), Vol. 2, pp. 273-322.

[26] P. A. R. Ade et al. (Planck Collaboration), Planck 2013 results. XXV. Searches for cosmic strings and other topological defects, Astron. Astrophys. 571, A25 (2014).

[27] A. Vilenkin, Gravitational field of vacuum domain walls and strings, Phys. Rev. D 23, 852 (1981).

[28] J. R. Gott III, Gravitational lensing effects of vacuum strings - Exact solutions, Astrophys. J. 288, 422 (1985); W. Hiscock, Exact gravitational field of a string, Phys. Rev. D 31, 3288 (1985); B. Linet, The static metrics with cylindrical symmetry describing a model of cosmic strings, Gen. Relativ. Gravit. 17, 1109 (1985); D. Garfinkle, General relativistic strings, Phys. Rev. D 32, 1323 (1985).

[29] M. E. X. Guimarães and B. Linet, Scalar Green's functions in an Euclidean space with a conical-type line singularity, Commun. Math. Phys. 165, 297 (1994).

[30] Yu. A. Sitenko and N. D. Vlasii, Induced vacuum energymomentum tensor in the background of a cosmic string, Classical Quantum Gravity 29, 095002 (2012).

[31] Handbook of Mathematical Functions, edited by M. Abramowitz and I. A. Stegun (Dover, New York, 1972).

[32] S. Bellucci, A. Saharian, and V. Bardeghyan, Induced fermionic current in toroidally compactified spacetimes with applications to cylindrical and toroidal nanotubes, Phys. Rev. D 82, 065011 (2010).

[33] A. P. Prudnikov, Yu. A. Brychkov, and O. I. Marichev, Integrals and Series (Gordon and Breach, New York, 1986), Vol. 2.

[34] I. S. Gradshteyn and I. M. Ryzhik, Table of Integrals, Series and Products (Academic, New York, 1980). 